\title{
MOAB B-3-CHX-A-DTPA
}

National Cancer Institute

\section{Source}

National Cancer Institute. MOAB B-3-CHX-A-DTPA. NCI Thesaurus. Code C29207.

A monoclonal antibody B3, recognizing the tumor-associated Lewis $Y$ antigen, is

conjug ated to a backbone-substituted derivative of DTPA, 2-(p-isothiocyanatobenzyl)cyclohexyl-diethylenetriaminepentaacetic acid (CHX-A DTPA). DTPA is a bifunctional chelating agent thereby allowing for the binding of radioisotopes to the immunoconjugate. 